\title{
Computer-aided Vacuum Cleaner Design
}

\author{
Hu Wenfei
}

Wenzhou Vocational \& Technical College, Wenzhou. 325035

61230934@qq.com

Keywords: Vacuum cleaner; design; user-friendly; psychology.

\begin{abstract}
In the context of traditional vacuum cleaner, this paper explores and discovers people's daily demand for vacuum cleaner, and explores a feasible plan which is more consistent with the demand of contemporary family life through analyzing and studying its evolutionary process, demand conditions of various families as well as combing with user-friendly design, taking full advantage of ergonomics in industrial design, and combing with relevant theories of design psychology and other industrial designs. Multi-functional intelligent vacuum cleaner can not only replace us to do some routine cleaning work, but also improve pleasure in life as well as quality of life. Wherein, the key technologies are: sensing technology, path planning technology, vacuuming technology and power technology, etc. With the quickening pace of life, intelligent vacuum cleaner can extricate us from tedious daily cleaning activities.
\end{abstract}

\section{Status and positioning}

\subsection{Development status of vacuum cleaner}

With continuous progress of the times as well as continuous improvement of people's minds, such situation that vacuum cleaner was once a luxury good for Chinese has changed. On the contrary, it gets rid of the former thinking that we cannot use the vacuum cleaner unless we need to clean the carpet and goes into the millions of households. While in nowadays, all the grounds might be performed routine cleaning with a vacuum cleaner. As a result, many ordinary families have a greatly increased demand for vacuum cleaner which reflects its larger market space from the side.

Contemporary lifestyle has changed and people are paying more attention to health and life quality. Consumer attitude of contemporary society differs from the past which is transferring from kneeing to enhance the living standards to improve the quality of life. In addition, our lifestyle is also changing from meet basic necessities of life to focus on scientific and technological content. Although people's lives have been improved, with the improvement of industry as well as economy, environmental pollution is becoming increasingly serious in recent years, and people's health needs to be improved and we have a urgent demand for a healthy and clean living environment. As a result, the vacuum cleaner goes into the lives of thousands of families.

Following washing machine and refrigerator, increasing development has made vacuum cleaner become the third large electric product. In addition, it is also listed in national planning statistics and development projects. It can be obviously seen that vacuum cleaner has a huge market competitiveness and demand, and it gradually occupies an indispensable position in our daily life.

When taking a broad view on various domestic as well as foreign markets, we can easily find that vacuum cleaner market is still not hot, but it does not simply mean small demand. On the contrary, it is full of opportunities. Just like a piece of cake which is increasingly perceived by people, vacuum cleaner more and more accepted by the public and our lives also become increasingly dependent on it. Whether its shape and color, or various materials all meet individual demands from the heart. Simply talking from the appearance, changeable bionic will certainly attract your attention which is colorful and may meet the demands of various family decorations. In material terms, it is created like industrial art. 


\subsection{Design positioning}

Multi-functional intelligent vacuum cleaner I design this time is mainly aiming at the shortcomings of some ordinary vacuum cleaners like single function, high noise and small clean range. It is designed with unique shape which is simple and elegant, and it is equipped with the function of intelligent return charging as well as intelligent watering. I pursue the unity of function and shape that meet the currently most popular fashion style, in addition, I also combine with the most popular design concept to design a convenient and intelligent vacuum cleaner. This vacuum cleaner adopts mechanical automatic steering principle and it will cut off the power automatically if there is malfunctions and caton phenomenon. Its cost is far lower than other products in the market.

\subsection{Design content}

Main design elements of multi-functional intelligent vacuum cleaner

1) Carry out market research, data check, patent look-up and other works about vacuum cleaner.

2) Working principle and motion analysis of vacuum cleaner.

\section{Modeling design}

\subsection{Basic modeling design of multi-functional intelligent vacuum cleaner}

Product modeling refers to a basic terminology that describes an item as well as its entire appearance. The purpose of product design is not just pursue pretty appearance, making the operation become easy and simple to meet the needs in their hearts and improve their spiritual pursuit is more important. It applies the aesthetic into various fields from visual communication design to product design. There is no doubt that shape is the most important factor in industrial design, and excellent shape design may make the product to be used with maximum efficiency and give a strong visual impact and impress. I use bionics principle to analyze and research biological shapes in the nature, and trigger my design inspiration and innovative thinking by means of the shape characteristics. Similar to our human beings, product shapes are also different with each other. As a result, as shown in Figure 1, I give full play to my imagination to re-innovate and reform the product modeling and then improve and enhance the value of product itself. Different design products present different states and shapes, the color and shape impress us firstly when recognizing a product. Therefore, the designer need to reflect various emotions of consumers in his design and allow users feel certain emotional resonance to achieve design purposes.
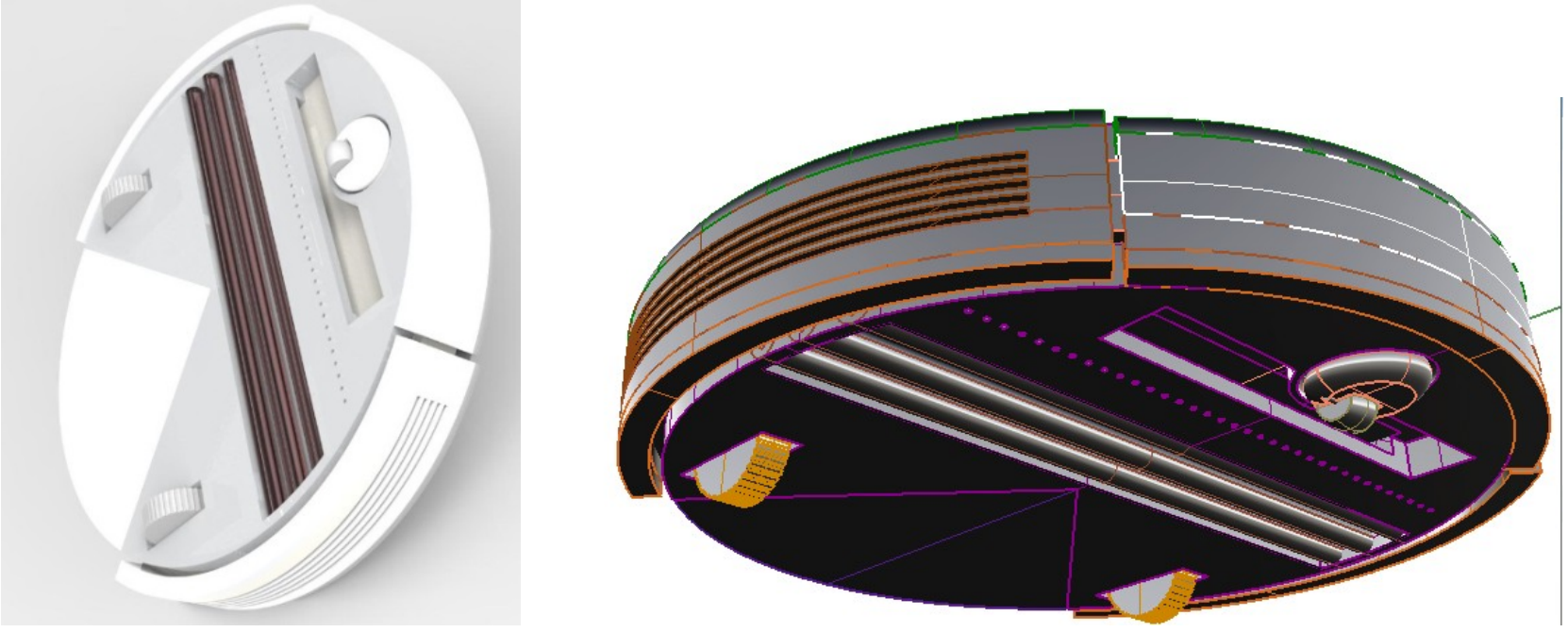

Figure 1 side and bottom design of vacuum cleaner

\subsection{Automatic steering design of multi-functional intelligent vacuum cleaner}

It consists of prime mover system, transmission system, work systems and control system and makes full use of mechanical automatic steering principle. Put vacuum cleaner on the floor, press the start button, and it will automatically rotate to avoid when encountering obstacles. In addition, it will 
automatically cut off the power and alert if there is malfunctions and caton phenomenon. The cost of technology is far lower than other vacuum cleaners in the market.

\subsection{Features of multi-functional intelligent vacuum cleaner}

Functions of intelligent vacuum cleaners on the market are relatively simple and they can only do some routine cleaning works like hair, dust and small objects on the ground, and some cleaning is beyond normal intelligent vacuum cleaners. Multi-functional intelligent vacuum cleaner not only equips with the features that most intelligent vacuum cleaners on the market have, but also has other main features. First: absorbent, we always encounter water or other liquid in daily cleaning and general vacuum cleaners will paralyze in such situation, while Multi-functional intelligent one may avoid absorbed liquid meet the motor and achieve the purpose of removing water. Second: scoop out some dirt which is difficult to clean. For example: most people choose to use a knife to scrape away dirt on home ground little by little which is time-consuming and laborious, while brushes on multi-functional intelligent vacuum cleaner may quickly resolve this issue. Third: the noise is as low as $50 \mathrm{~dB}$. Forth: clean some daily invisible rubbishes like wax worm. These are common but troubling in our daily life, in order to solve these problems, multi-functional intelligent vacuum cleaner is the only choice.
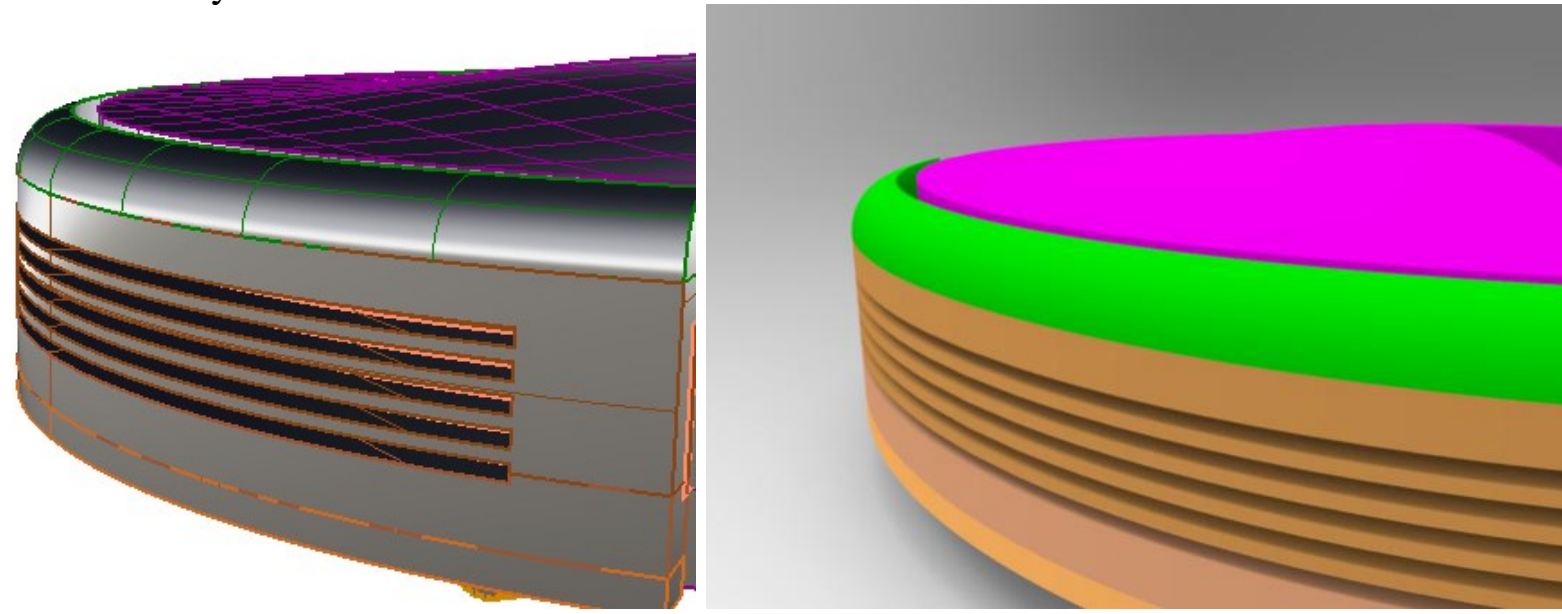

Figure 2-4-1 Dust box and vent behind of vacuum cleaner

\subsection{Basic parameters of multi-functional intelligent vacuum cleaner}

Because of the special working environment, vacuum cleaner tends to compact. And the designed one round appearance with $20 \mathrm{~cm}$ radius and $12 \mathrm{~cm}$ height.

\section{Structure of multi-functional intelligent vacuum cleaner}

\subsection{Product material selection}

Production is inseparable from material and in addition, material is the basic existing form of product design and real product can not be separated from material to exist. Different materials give different feelings to us, visual feature of the product is just the feature that such material brings to us when applying into a product. As a result, quality of the material is crucial for products.

Different materials have different textures and similarly, different materials give us different feelings about cold and worm as well as hard and soft. As a result, I choose ABS plastic for this product and its structural formula is shown in Figure 3. This material has a high wear resistance and equips with texture and beauty at the same time.

Various materials all have their own textures and beauties. In order to show the shape beauty through using good materials, we need to understand the properties and features of various materials to complete product design. 


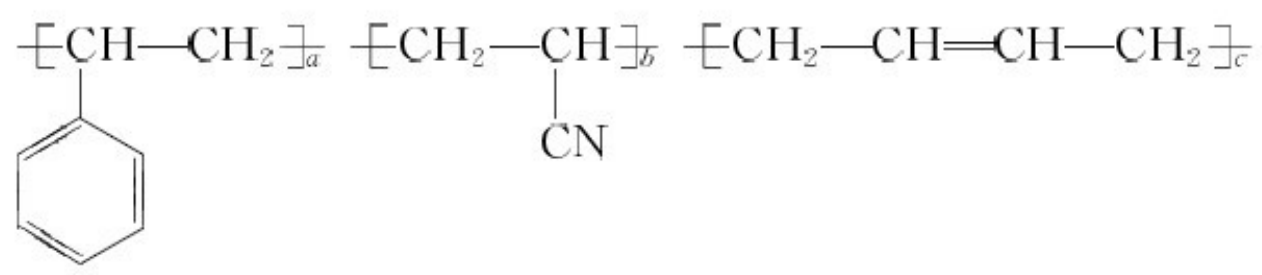

\subsection{Product color selection}

Figure 3 ABS resin structure

The world we live is colorful, different items have different colors and presents different visual effects. If we say material is an important part to the product, color is an integral part. Many information about the product are all conveyed through colors which have a special impact on our feeling. Reasonable use of color may play a "finishing touch" role.

\section{Summary}

There are always corners and dirt which are difficult to clean when doing housework, and it really brings a lot of troubles to us. Traditional vacuum cleaner can only clean up some visible dust, hair and other debris, while such intelligent vacuum cleaner is different, its flexible cutting tool and powerful vacuum absorption will clean up all the things that beyond common vacuum cleaner. It has high superiority because of the powerful features such as: intelligent return charging and sprinkler cleaning. With the continuous development of intelligent life, many invisible dirt all need to be cleaned, but ordinary vacuum cleaner can not do these. As a result, researching and developing a multi-functional intelligent vacuum cleaner is one of the possible options to solve the issues we mentioned above, and it effectively solve the problems like single feature, noise, cleaning range and species and has a far-reaching significance. I also analyze the advantages and disadvantages of multi-functional intelligent vacuum cleaner, and evaluate both domestic and foreign development status. In addition, I also design a new type of intelligent vacuum cleaner which may clean all the dust, hair and other dirt, and introduces its main components which includes operation, design and working principle.

\section{References}

[1] PI Yong-sheng.Product Semantic Design[M].Chongqing: SouthwestChina Normal University Press, 2009.

[2] RUDOLF Arnheim.Art and intuition[M].TENG Shou-yao, Translate.Beijin: China Social Sciences Press, 1984.

[3] YANG Jun-shun, MA Lei, YANG Gang.Non-material Designof Product and Sustainable Development[J].Packaging Engineering, 2006, 27 (3) : 153-155.

[4] JIANG Jin-chen, PI Yong-sheng.Principles and methods of product design[M].Chongqing: Southwest China Normal University Press, 2009.

[5] PI Yong-sheng.Recognition of the role of industrial design[J].Zhuangshi, 2010 (10) : $129-130$.

[6] WANG Shou-zhi.History of Modern Design in the World[M].Shengzhen : New Century Press, 1995. 